\title{
Instantánea Urbana: Rudimentos Analíticos para una Aproximación Fenoménica Sistemática al Estudio del Comportamiento de Nuestro Campo Visual en Relación a la Percepción de Personas en el Espacio Urbano
}

\author{
Urban Instant: Analytical Rudiments for a Systematic Phenomenological Approach to the Study of our \\ Visual Field Behavior in Relation to People Perception in Urban Space
}

\author{
Claudio Araneda \\ Univesidad del Bío-Bío, Chile \\ claraneda73@gmail.com
}

\begin{abstract}
Desde la publicación casi simultánea de los manifiestos urbanos de Jane Jacobs (1961) y Kevin Lynch (1960), la diciplina urbana ha sido permeada de forma creciente por el impulso fenomenológico. Si bien comparten principios metodológicos, representan aproximaciones distintivas a los estudios urbanos; dos linajes de pensamiento urbano cuya distinción constituye el punto de partida de esta investigación. Su objetivo fundamental es el de contribuir al desarrollo metodológico del linaje de Jacobs. Con este fin se esbozan rudimentos analíticos que permitirían el comienzo de un estudio sistemático de los patrones de presencia de personas en nuestro campo visual en el espacio urbano. Este trabajo constituye parte de los resultados del proyecto fondecyt de iniciación 11110450.
\end{abstract}

Keywords: Fenómeno urbano; Fenomenología; Space syntax; Jane Jacobs; Concepción.

\section{Introducción}

El argumento central de este paper conecta con la crítica fundamental de Hillier (1996) respecto a la tendencia histórica y atávica del urbanismo a prescribir dogmáticamente así como a su dramática carencia de herramientas de análisis urbano. Conecta también con la crítica efectuada por Ratti $(2004,2005)$ al trabajo de Hillier respecto al salto de fe implícito en las predicciones de Space Syntax basadas en el mapa axial. Difiere con ambos, sin embargo, en cuanto a objeto de estudio al cual se aplican. Argumenta que a pesar del gran progreso realizado por estas exitosas técnicas de análisis derivadas del linaje de Lynch estas son eminentemente especulativas en tanto ninguna de ellas aborda el problema de la percepción de gente (de carne y hueso) en el espacio urbano en primera persona.

Como resultado de esto, las predicciones relativas a los patrones de presencia de gente en la ciudad han permanecido susceptibles a error pues no proceden de la observación directa del objeto de estudio en cuestión sino que de especulaciones a priori derivadas de algoritmos computacionales. A la luz de esto, el objetivo fundamental de esta investigación es el desarrollo de rudimentos analíticos para una aproximación experiencial, en primera persona, al estudio de la percepción de seres humanos en el espacio urbano, distinguiendo a estos- no al espacio- como nuestro objeto de estudio. Con esto, contribuir al desarrollo del linaje de Jacobs, actualmente rezagado.

\section{Distinguiendo el Linaje de Lynch del Linaje de Jacobs}

Mientras Lynch se abocó al estudio de la percepción del espacio urbano durante nuestras navegaciones por la ciudad, Jacobs se concentró en la percepción de gente durante las mismas. Es decir, si bien compartieron método, discreparon en cuanto a objeto de estudio. Esta distinción resulta fundamental para entender el urbanismo contemporáneo pues constituye la semilla de dos escuelas de pensamiento crítico. Una de corte arqueológico/estructural que busca el conocimiento urbano en el estudio de la ciudad construida (el espacio urbano). Otra, de corte antropológico/sistémico, que lo busca en el estudio de las relaciones humanas, un objeto de estudio elusivo y difícil de estudia dado su carácter de blanco en movimiento. A la primera escuela la denominamos como el linaje de Lynch. A la segunda como el linaje de Jacobs. Si bien el trabajo de Jacobs (1961) entronca directamente con las investigaciones pioneras en Proxemia de E.T. Hall $(1969,1973,1976)$ y en tanto tal con las investigaciones de Jan Gehl (2001, 2010), Bryan Lawson (2001), Herman Hertzberger (1991), Jaime Garretón (1975) y puede incluso ser rastreado hasta el desconocido trabajo del arquitecto alemán Herman Maertens (1884), ha permanecido analíticamente débil.

El linaje de Lynch, por su parte, ha experimentado un desarrollo sostenido. Fruto maduro lo constituye el paradigmático trabajo 
desarrollado por el Space Syntax Lab quienes desarrollaron una herramienta de alta precisión para el estudio topológico de plantas arquitecturales y urbanas descubriendo una constante entre ellas y los patrones de circulación peatonal y presencia de gente en las calles de una ciudad. Otro brote fértil ha sido el trabajo desarrollado por Carlo Ratti en el Senseable Lab del MIT, quienes llevando el impulso sintáctico espacial hasta sus últimas consecuencias desarrollaron modelos digitales de elevaciones (DEM) con miras a complementar la reduccionista aproximación bidimensional topológica (Ratti, 2005). En paralelo, han desarrollado sofisticadas técnicas de rastreo de señales telefónicas que proporcionan una nueva lectura de los patrones de presencia de gente en la ciudad (Calabrese et.al., 2007a, 2007b, 2007c). Por último, Batty (1998) se ha concentrado en el desarrollo de modelos basados en agentes (ABM) para el estudio de los patrones de uso y movimiento del espacio urbano por parte de los peatones. Esto, por mencionar algunos casos representativos. El impulso puesto en marcha por este linaje y en particular por el trabajo de Lynch siguen dando frutos (Morello \& Ratti 2008).

\section{Crítica Constructiva a la Aproximación Arqueológico Estructural}

Todos los casos arriba mencionados se inscriben en el paradigma del diseño basado en evidencia promulgado por Hillier. Sin embargo, todos comparten el siguiente rasgo metodológico. Cuando de registrar, procesar y diagramar información en forma de señales telefónicas electromagnéticas o bien, en forma de topología y morfología se ha tratado, han procedido con el mayor rigor fenomenológico. Es decir, han extraído el conocimiento de una relación directa con el objeto de estudio que los ocupa. Sin embargo, cuando de registrar, procesar y diagramar información en forma de personas se ha tratado, se han comportado de forma laxa y complaciente. El proceso de registro se reduce a conteos de personas y este a su vez en tablas y gráficos de modo que ni el proceso de observación directa ni la información en forma de personas propiamente tal logra ser visualizado.

Si estamos de acuerdo en que el zeitgeist de la época en análisis urbano es el conocimiento basado en evidencia, entonces, técnicas igualmente precisas y sofisticadas para un estudio fenoménico de la información en forma de personas deben ser desarrolladas. Con este fin, esta investigación propone una nueva forma de registro organizado de los patrones de conducta de nuestro campo visual (fotográficamente abstraído) en relación a la percepción de información en forma de personas. Un nuevo "layer" que podrá ser luego cotejado con la información topológica, complementando la hasta ahora abstracta aproximación a los patrones de presencia de gente en la ciudad. Lo llamamos "instantánea o polaroid urbana” (Araneda, 2013).

\section{Instantánea Urbana (Un esbozo metodológico)}

Aceptando de entrada que todo registro de una experiencia es una reducción de ella, proponemos lo siguiente. Así como los cálculos topológicos efectuados por medio del mapa axial dependen de la distancia de cada línea respecto a todas la otras líneas del área bajo escrutinio, para obtener lecturas fenoménicas exactas sobre los patrones de presencia de gente en las calles en cualquier momento determinado, necesitamos capturar el estado de nuestro campo visual respecto a la percepción de personas en todas las calles contenidas dentro del mismo perímetro de forma simultánea. Es decir, una instantánea urbana.

Si bien lógica en su explicación, la implementación de esta aproximación plantea interesantes problemas prácticos. Por ejemplo, si la intensidad necesaria para validar la precisión de un estudio es de, por ejemplo, 3 fotografías por segmentos (o bien, por manzana) y la calle en cuestión esta compuesta de 10 segmentos o manzanas, el total de fotografías necesarias para esta calle será de 30. Esto significa que si lo que queremos es obtener una verdadera polaroid de esta calle, se necesitan 30 distintas cámaras; 30 personas o máquinas haciendo "click" exactamente al mismo tiempo y bajo el mismo formato (misma altura, dirección, apertura de lente, etc).

Ahora bien, si el numero total de calles dentro del área escogida es de, por ejemplo, 20, la cantidad de tomas necesarias para obtener la instantánea urbana sube a 600 ! Como logísticamente este método es engorroso y costoso en términos de recursos humanossi bien no imposible- en el caso analizado recurrimos a las navegaciones Lyncheanas. Es decir, viajes video/fotográficos a lo largo de todas las calles bajo análisis. La consistencia y confiabilidad de esta aproximación dependerá exclusivamente de la cantidad de viajes realizados por día, mes y año, por cada calle bajo observación.

\section{Caso de Estudio (Concepción)}

Todas las calles del perímetro histórico de la ciudad de Concepción son recorridas de forma lineal (itinerarios por viaje en Figura 1). Con este fin, creamos una patrulla de registro fotográfico conformada por 26 estudiantes.

El formato estableció que todas las calles serían recorridas en línea recta de un extremo a otro del perímetro y que todos los viajes comenzarías a la misma hora. A menos que sea imposible, el itinerario debe ser hecho por áreas peatonales. Dependiendo de la cantidad de tomas, se obtiene un registro fotográfico de intensidad variable. Es decir, un numero " $n$ " de frames por segmento de calle, entendiendo por segmento en este caso, el tramo de calle definido entre intersecciones con otras calles o bien, entre esquinas.

En este caso, utilizamos una intensidad baja de 2 fotos por segmento (manzana). Organizadas en formato de filmstrip, este registro crudo y no editado muestra el estado general del comportamiento de nuestro campo visual durante los viajes urbanos. 


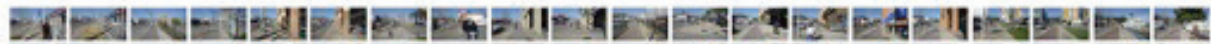

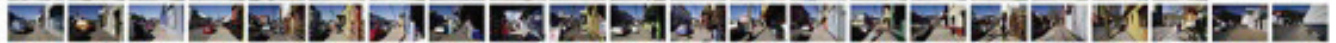

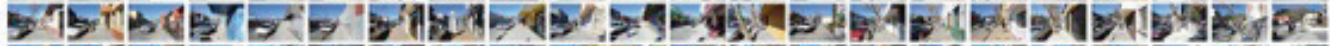

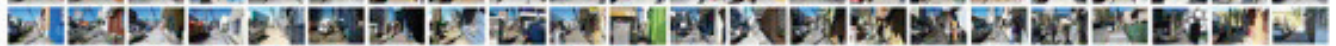

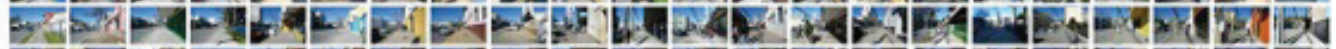

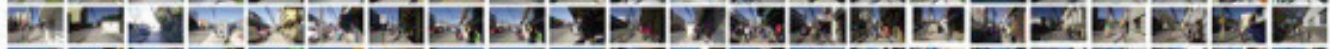

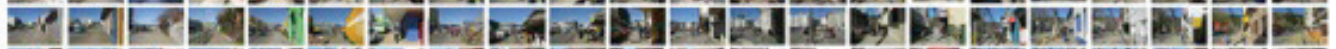

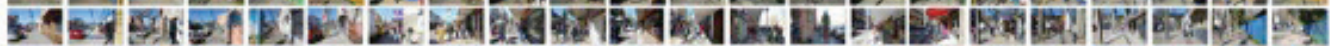

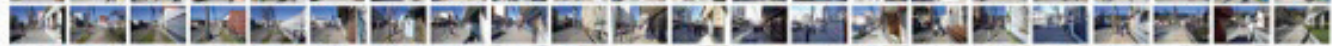

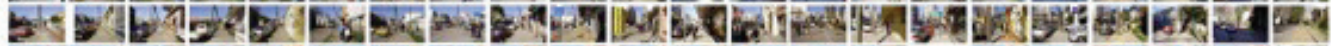

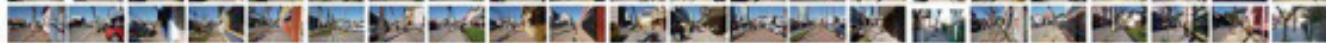

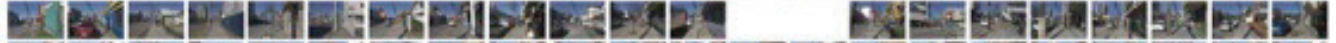

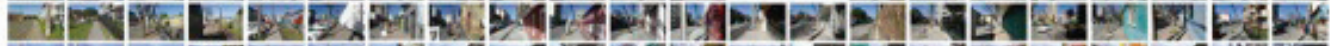

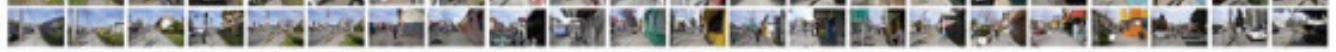

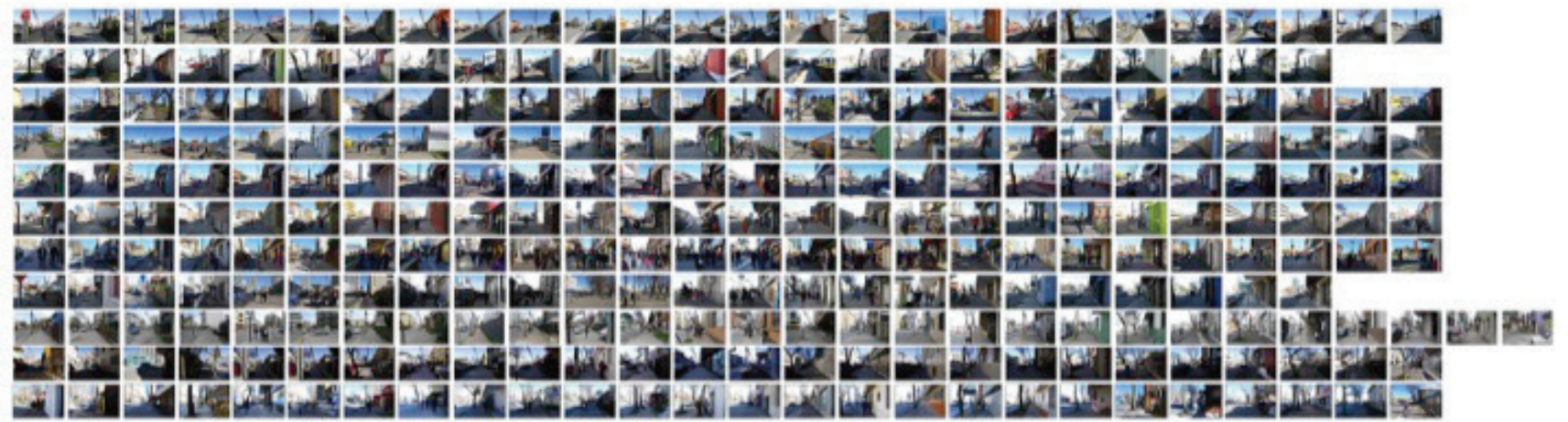

Figura 1: Filmstrip fotográfico de navegaciones transversales (arriba) y longitudinales (abajo).

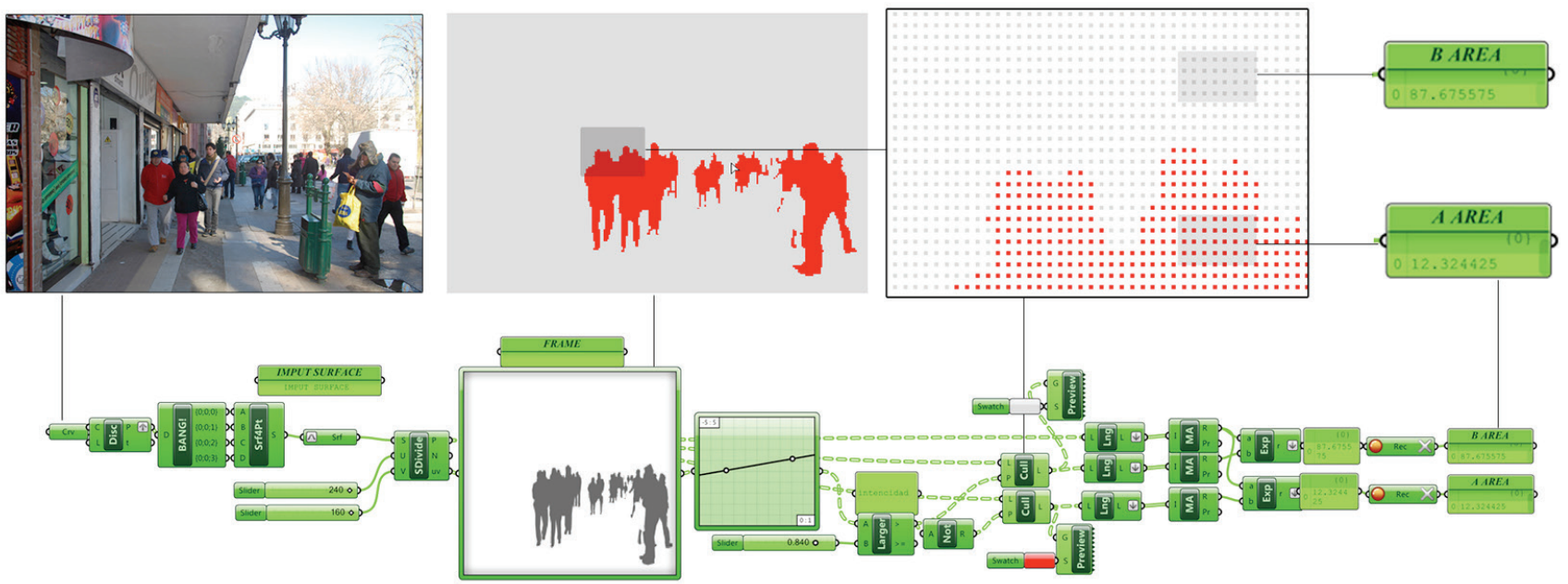

Figura 12 Proceso de rasterización y cálculo de información en forma de personas.por frame. 

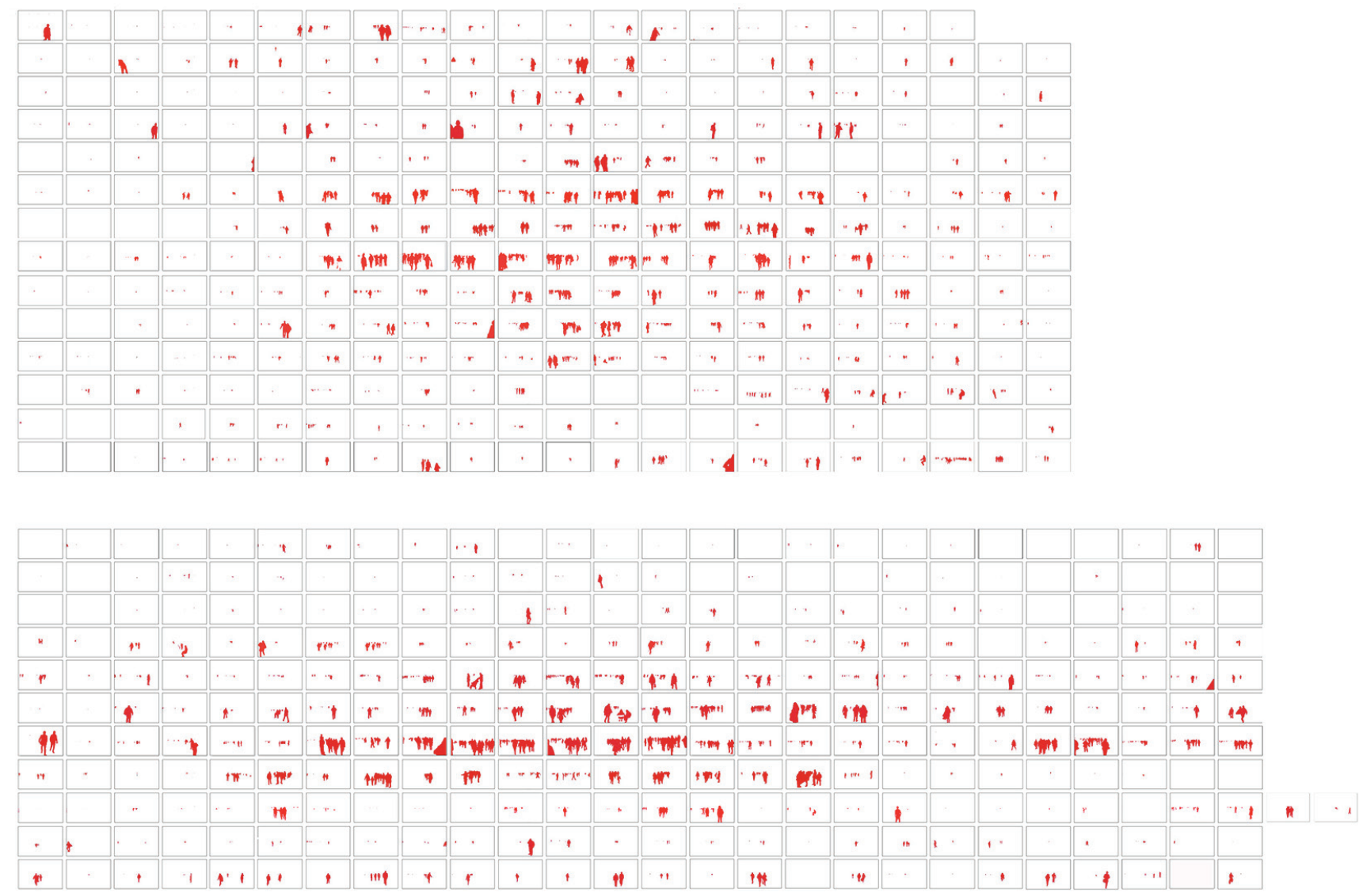

Figura 3: Filmstrips rasterizados con información en forma de personas.
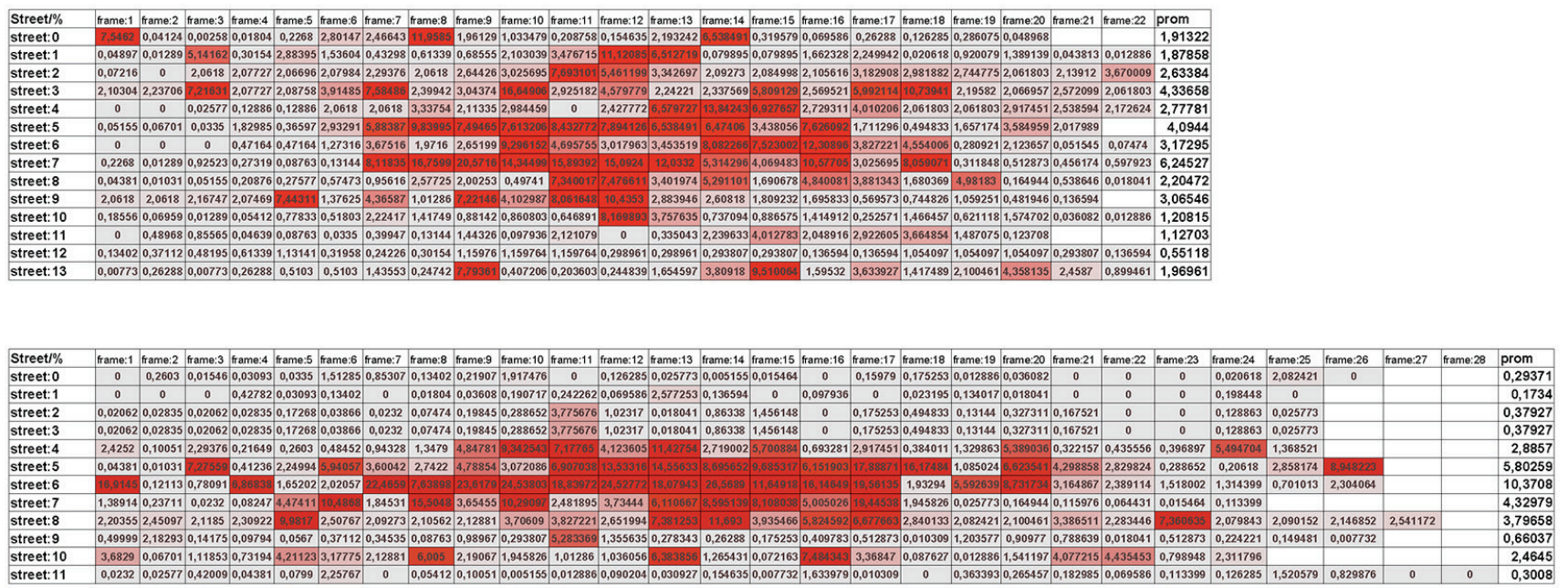

Figura 4: Tabla con cantidades de informacion visual en forma de personas por captura (columnas) y promedios por calle (filas).

Aplicando gráfica raster simple, procedemos a transformar toda la información en forma de personas en superficies coloreada por foto. (Figura 2 \& 3). Esto revela cuanta superficie de nuestro campo visual (fotográficamente abstraído) esta poblada de información en forma de personas. Comparando el área coloreada con el área total del frame, obtenemos el porcentaje de información en forma de gente para ese frame en particular. La misma operación para todos los frames de una misma calle arroja como resultado el porcentaje promedio de información en forma de personas. Finalmente, las misma operación para todas las calles del área analizada da como resultado el promedio de información en forma de personas para esta ciudad en particular (Figura 3). 


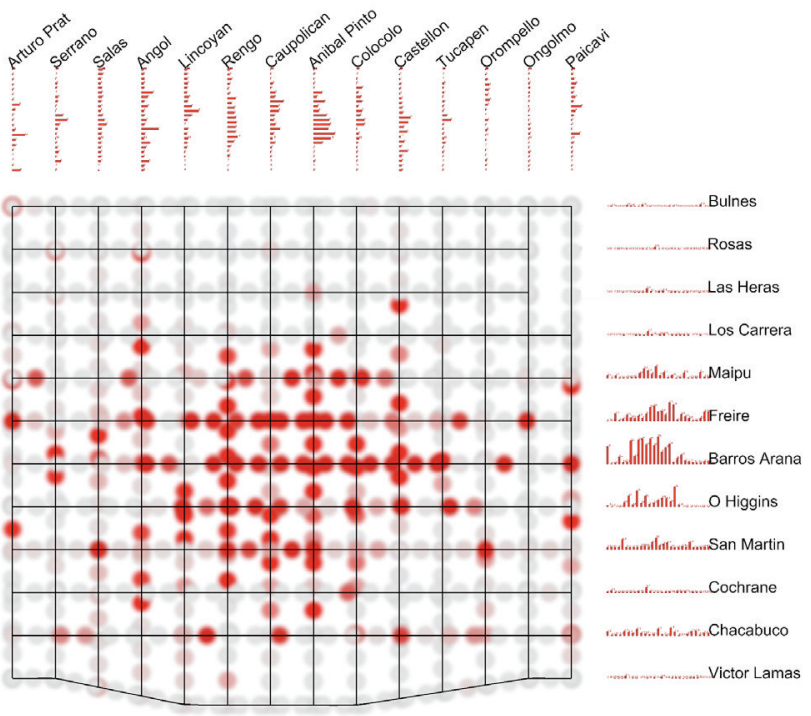

Figura 5: Sintaxis bidimensional de los patrones de presencia de gente en nuestro campo visual.

\section{Conclusiones}

A diferencia de las técnicas de sintaxis espacial, las que predicen donde existe mayor probabilidad de circulación urbana, la metodología aquí expuesta revela ipso facto donde la gente se encuentra de forma efectiva. Es decir, al partir desde la experiencia, la instantánea urbana reduce a cero la especulación implícita en la mayoría de las herramientas de análisis desarrolladas por los representantes del linaje de Lynch. Para el caso analizado, la representación planimétrica bidimensional muestra una notable similitud con la representación gráfica utilizada por space syntax (Figura 4) el que, si fuese aplicado localmente, revelaría las calles centrales del área analizada como las mas integradas. Pero esta coincidencia ha probado no ser constante. De este modo, si bien la información obtenida por ambas vías puede ser cuantitativamente afín, difieren ampliamente en cualidad: una muestra potencial, la otra actualidad; una revela información de la ciudad construida, la otra información de la ciudad por construir. Esto puede resultar crucial en aquellos casos en donde las predicciones basadas en el mapa axial no se corresponde con la realidad de una determinada calle o área urbana ofreciendo un complemento fenoménicamente exacto, gráficamente elocuente y académicamente didáctico. Al mimo tiempo, ayuda a explicar la no infalibilidad de uno de los argumentos mas controversiales de Hillier, a saber, que el factor determinante tras el fenómeno de atracción urbana es la configuración espacial (Hillier, 1999, page 06.20). En suma, no todo está escrito aun en análisis urbano.

\section{References}

Araneda, C. (2013). The Jacobs' Urban Lineage Revisited. Analytical rudiments for the further development of the phenomenological approach to the study of the perception of people in urban space implicit in Jane Jacobs' work, Proceedings of the eCAADe Conference, Delft, Netherlands, Vol.2, pp. 355-363.

Batty M, Jiang B, Thurstain-Goodwin M. (1998). 'Local movement: agent-based models of pedestrian flow", WP 4, Centre for Advanced Spatial Analysis, University College London, http://www.casa.ucl.ac.uk

Calabrese, F., Kloeckl, K., and Ratti, C. (2007a). 'WikiCity: Real-Time Location- Sensitive Tools for The City'. Proceedings of the 10th International Conference on Computers in Urban Planning and Urban Management (CUPUM07). [online]. Available at: http://senseable.mit.edu/papers/pdf/2007_Calabrese_et_al_Wiki City_CUPUM.pdf

Calabrese, F., Kloeckl, K., and Ratti, C. (2007b). 'WikiCity: Connecting the Tangible And the Virtual Realm of a City'. Geolnformatics, 10(8), 42--45. [online]. Available at: http://senseable.mit.edu/papers/pdf/2007_Ratti_et_al_WikiCity_ Geolnformatics.pdf

Calabrese, F., Colonna, M., Lovisolo, P., Parata, D., and Ratti, C. (2007c). 'Real- Time Urban Monitoring Using Cellular Phones: a Case-Study in Rome'. [online] Available at: http://senseable.mit.edu/papers/pdf/2007_Calabrese_et_al_Rom e_Unpub.pdf

Garretón, J. (1975). Una Teoría Cibernética de la Ciudad y su Sistema. Ediciones Nueva Visión, Buenos Aires, Argentina.

Gehl, J. (2001). Life Between Buildings. Using Public Space, The Danish Architectural Press, Denmark.

Gehl, J.. (2010) Cities for People. Island Press, USA.

Hall, E.. (1969). The Hidden Dimension, Anchor Books Editions, USA

Hall, E. (1973). The Silent Language, Anchor Books Editions, USA

Hall, E. (1976). Beyond Culture, Anchor Books Editions, USA.

Hertzberger, H. (1991). Lessons for Students in Architecture. 010 Uitgeverij, Netherlands.

Hillier, B. (1996). Space is the Machine, Cambridge University Press, UK.

Jacobs, J. (1961). The Death and Life of Great American Cities. The Failure of Town Planning, Penguin Books, UK.

Lawson, B. (2001). The Language of Space. The Architectural Press, Oxford, UK.

Lynch, K. (1960), The Image of the City, MIT Press, Cambridge MA.

Maertens, H. (1884), Der Optsiche Masstab in den Bildenden Kuensten, Wassmuth, 2nd edition, Berlin.

Morello, E \& Ratti, C. (2008). 'A Digital Image of the City: 3-D Isovists and a Tribute to Kevin Lynch'. [online]. Available at: http://senseable.mit.edu/papers/pdf/2008_Morello_Ratti_Enviro nment\%20and\%20Planning\%20B.pdf

Mumford L \& Miller D (ed.), 1986, 'The Lewis Mumford Reader'. Pantheon Books, USA.

Ratti C. (2005). 'The lineage of the line: space syntax parameters from the analysis of urban DEMs', Environment and Planning B: Planning and Design 32(4) 547 - 566 